\title{
LOS SALTAMONTES (Orth.: Acridoidea) DE SIERRA ESPUÑA (Murcia, SE. ESPAÑA): TIPIFICACION DE SUS POBLACIONES
}

\author{
Por \\ M. ${ }^{a}$ D. García ${ }^{1}$; J.J. Presa ${ }^{1}$ y L. Ramírez-Díaz ${ }^{2}$ \\ QUANTITATIVE AND ECOLOGICAL STUDY OF SOME \\ GRASSHOPPERS POPULATION (Orth.: Acridoidea) \\ IN SIERRA ESPUÑA (MURCIA, SE. ESPAÑA)
}

\section{SUMMARY}

Using some different statistical techniques of multivariate analysis, mainly Binary Discriminant Analysis (BDA), ecological groups of grasshoppers populations have been defined and related to the main physiognomic vegetation types of Sierra Espuña.

The relationships between species have also been studied and it has been confirmed that the type of vegetation is a fundamental factor in determining the distribution of populations and communities of Acridoidea.

Results are summarized in a comprehensive table that shows the preferences of grasshoppers populations and vegetation.

\section{RESUMEN}

Utilizando diferentes técnicas estadísticas de análisis multivariante,, entre ellas y de manera destacada el análisis binario discriminante ( $(\mathrm{BD} A »$, Binary Discriminat Analysis), se definen grupos ecológicos de poblaciones de saltamontes en relación con los tipos fisionómicos de vegetación de mayor relevancia en Sierra Espuña.

Se estudian relaciones entre especies, y se confirma como fundamental el tipo de vegetación como factor ambiental determinante de la distribución de las poblaciones y comunidades de acrididos.

Se esquematizan los resultados de un cuadro-resumen de preferencias: poblaciones de saltamontes-vegetación.

(1) Departamento de Zoologia. Facultad de Biología. Universidad de Murcia.

(2) Departamento de Ecología. Facultad de Biología. Universidad de Murcia. 


\section{INTRODUCCION}

La distribución de las poblaciones de saltamontes y los factores que determinan esta distribución han sido objeto de atención de cierto número de autores que, tras sus estudios, han llegado a conclusiones dispares.

Por ejemplo, se han establecido relaciones entre la distribución de los ortópteros y la altitud, pisos de vegetación, acción conjunta de factores macro y microclimáticos, la estructura social de las poblaciones (BACCETTI, 1963; MARTY, 1961; GAJE \& MUKERJI, 1977; CLARIDGE \& SINGHRAO, 1978). Todos estos datos y otros han sido expuestos de modo resumido en PRESA et al., 1983. Estos mismos autores opinan que el estudio de la distribución de las comunidades de ortópteros debe basarse en un conocimiento preciso de la fisionomía, abundancia y patrones de distribución de la vegetación, así como de las propiedades físicoquímicas y nutritivas de la misma y no en la estratificación altitudinal de pisos de vegetación, exclusivamente.

El objetivo de este estudio consiste en la tipificación de las poblaciones de ortópteros de Sierra Espuña y la consiguiente delimitación de especies indicadoras (aspectos cenológicos y ecológicos).

El tratamiento de los datos se ha realizado mediante técnicas estadísticas de análisis factorial, también utilizadas en otros trabajos, referentes a zonas montañosas (PRESA et al., 1983), o no (DAHDOUH et al., 1978; DURANTON \& LECOQ, 1980).

Este tipo de trabajo, en la Península Ibérica, sólo se ha realizado con anterioridad en la Sierra de Guadarrama, con la que Sierra Espuña presenta una serie importante de diferencias, como su posición geográfica, la altitud absoluta y los pisos de vegetación (GARCIA, 1983).

Respecto al primer punto, Sierra Espuña reviste interés por constituir una isla de tipo climático continental mediterráneo, dentro del clima mediterráneo subdesértico que la rodea. En cuanto a la altitud absoluta, la que presenta Sierra Espuña es ostensiblemente inferior, aunque su rango es similar. Por último, en Sierra Espuña no aparece diferenciación altitudinal en pisos de vegetación. 
La zona de estudio comprende Sierra Espuña, situada en las estribaciones del Sistema Bético, limitada al Sur por la depresión del Guadalentín y al Norte por el Barranco de Malvariche, encontrándose relativamente aislada de otros sistemas montañosos.

Los aspectos morfoestructurales del territorio han sido revisados parcialmente por LILLO CARPIO, 1971; LOPEZ BERMUDEZ, 1975; NAVARRO ALVARGONZALEZ \& TRIGUEROS MOLINA, 1963; PEYRE \& PEYRE, 1960; VALENZUELA, 1963-64. Desde el punto de vista botánico destacan, fundamentalmente, el trabajo de ESTEVE CHUECA, 1972 y, más modernamente, lo que realiza el Departamento de Botánica de la Universidad de Murcia, aún inédito.

Para la obtención de los datos se planificó un programa de muestreo de dos modos diferentes, extensiva e intensivamente.

Con objeto de recoger información sobre la variación temporal de las taxocenosis de acrídidos, para estudiar su fenología, aspectos dinámicos, así como la faunística y sistemática de las diferentes poblaciones (GARCIA, 1983), la zona de estudio se muestreó de forma extensiva mensualmente, desde Septiembre de 1980 a Septiembre de 1982, en cuarenta estaciones repartidas espacialmente por todo el territorio estudiado (Fig. 1), cuya nominación se relaciona a continuación.

El estudio intensivo se realizó en 27 estaciones, que aparecen señaladas con $\left(^{*}\right)$ en la relación anterior, en las cuales se midieron 18 variables ambientales diferentes. Para más detalles sobre la caracterización ambiental de las estaciones de muestreo ver GARCIA, 1983.

Se recolectaron 35 especies en un total de 513 muestras en el conjunto del estudio (GARCIA, 1983).

Se realizaron estimas relativas de la densidad (SOUTHWOOD, 1978), básicamente por unidad de esfuerzo (MONTE \& RAMIREZ-DIAZ, 1978). Para ello se empleó una red entomológica de $50 \mathrm{~cm}$. de diámetro. Cada unidad de vegetación era mangueada intensivamente durante 30 minutos. Este método, en condiciones climáticas y técnicas similares, proporciona estimas fiables de la densidad (CLARK, 1947; DREUX, 1961; CLARIDGE \& SINGHRAO, 1978; VOISIN, 1980). 
1. Casa del Paleto*

2. Casa del Estrecho*

3. Morra de las Cuevas

4. Casa de Leiva*

5. Barranco del Canalón*

6. Los Carriles*

7. El Berro*

8. Loma del la Cueva del Lobo Rabote*

9. Barranco de la Fuente del Piojo*

10. Llano del Lavador*

11. Barranco del Sombrero

12. Huerta de las Labores*

13. Casa de las Labores*

14. El Morrón*

15. Pozos de la Nieve (prado)*

16. Pozos de la Nieve (pinar)*

17. Barranco del Gallego*

18. Collado de las Chaparras*

19. Barranco de los Frailes*

20. Campix*
21. Majal del Pajarillo*

22. Cabezo del Asperonal*

23. Collado del Pilón*

24. Collado Blanco*

25. Prado Mayor*

26. Barranco de Leiva*

27. Barranco de la Perdiz (pastizal)*

28. Barranco de la Perdiz (cortafuegos)*

29. Barranco de la Perdiz (pinar)*

30. Barranco del Jardal.

31. Cuestas del Perdigón

32. Valle del Río Espuña

33. Fuente del Sol

34. Barranco del Azud

35. .Fuente de la Madroñera

36. Collado de la Sabina

37. Casa Nueva

38. Poyos de Pedro López

39. Barranco del Pozo

40. Casa de la Carrasca

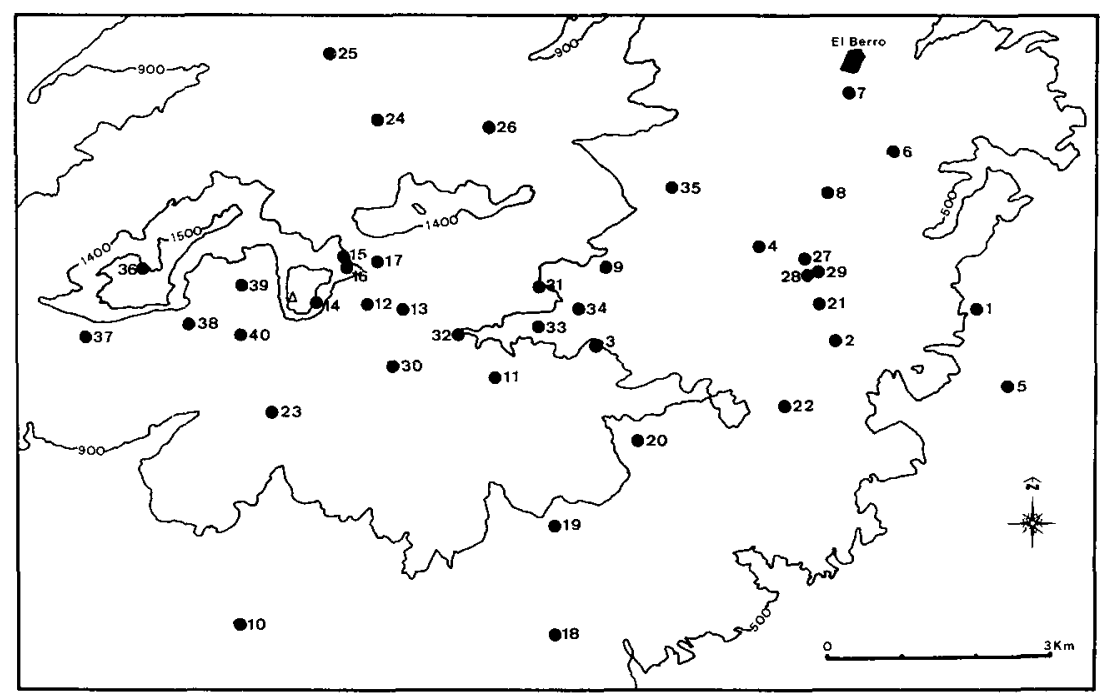

Figura 1. - Localizacion geogratica de las estaciones de muestreo consideradas en el estudio. 
Las estimas se completaron mediante conteo directo y rastreo de superficie (SOYER, 1965; PASCUAL, 1978).

La información obtenida se estudió mediante técnicas estadísticas multivariantes (PRESA et al., 1983).

\section{RESULTADOS}

Con objeto de llegar a conocer la relación entre las especies y poder caracterizar la estructura de la comunidad de saltamontes, se analizaron los datos a partir de una matriz de 28 especies y 324 muestras, resultado de eliminar de la matriz original (35 especies y 324 muestras del estudio intensivo de un año), las especies presentes únicamente en una estación de muestreo.

Esta matriz de datos se procesó mediante la técnica de «Factor Analysis» (BMDP4M), DIXON \& BROWN, 1979, de las siguientes formas: en primer lugar se consideró la totalidad de las muestras; en segundo lugar y con objeto de reducir la heterogeneidad temporal, se examinaron 52 muestras - resultado de agrupar las muestras en períodos de seis meses, eliminando dos semestres que no presentaron ninguna capturay 28 especies; en tercer lugar se examinaron 27 muestras (número total de estaciones) y 28 especies y, por último, una matriz de 24 especies - ya que los análisis indicaron la conveniencia de eliminar las especies de distribución restringida- y 27 muestras.

En todos los casos aparecieron grupos de especies poco definidos y cuya interpretación ecológica presentaba problemas por las peculiaridades de la estructura de las distintas matrices, principalmente por la existencia de un gran número de ceros (GARCIA, 1983).

A fin de que el componente cuantitativo, abundancia de individuos, no enmascarase las posibles relaciones especies-medios, se aplicó el análisis factorial de correspondencias, en el que sólo se hizo constar la presencia o ausencia de una especie en una determinada muestra. En primer lugar se aplicó a una matriz de dimensiones 28 especies y 52 muestras (ver más atrás). En segundo lugar, con objeto de reducir la variabilidad temporal, se analizó una matriz de 28 especies y 27 muestras. 
Los resultados de estos análisis mostraron una tendencia a agrupar a las especies en función de los tipos fisionómicos de vegetación (GARCIA, 1983) (Fig. 2).

Para resaltar estas relaciones se utilizó el análisis binario discriminante (BDA), que permite determinar las relaciones especie-medio al ordenarlas en un espacio multidimensional (forma Q) o al formar grupos de especies con patrones ecológicos similares con respecto a la variable ambiental que se estudie (forma R) (STRAHLER, 1978, PRESA et al., 1983).

Como se indicó anteriormente, se consideró un único factor o variable ambiental, los tipos de vegetación; siete estados según la fisionomía y estr to de sucesión de la vegetación, que fueron: Vegetación bajo clara (VEBARA); Pastizal agostante alto (PATALO); Pastizal no agostante bajo (PANOBA); Pastizal no agostante alto (PANOAL); Matorral almohadillado (MATOAL); Matorral alto claro (MATORO) y Matorral alto denso (MATODE).

Para realizar este análisis se tuvo en cuenta la totalidad de las estaciones muestreadas (40) temporalmente en su conjunto e incluyendo otros muestreos no localizados en el espacio o en el tiempo.

\section{ANALISIS BINARIO DISCRIMINANTE PARA LA VARIABLE TIPOS DE VEGETACION: 51 MUESTRAS Y 30 ESPECIES}

Unicamente 19 especies resultaron significativas a un determinado tipo de vegetación para $\mathrm{p}<0,05$ al ser evaluadas mediante el estadístico G (PRESA et al., 1983). Los residuos estandarizados para estas especies fueron sometidos al «Factor Analysis» en las formas $\langle Q\rangle y \ll R »$.

La tabla 1 recoge los factores de carga rotados de los siete tipos de vegetación en los tres primeros ejes y la figura 3 representa las 19 especies en el plano definido por los ejes I y II (forma Q).

En este primer análisis aparece claramente segregado el Matorral almohadillado (MATOAL) y los resultados del mismo indican la conveniencia de repetir el análisis sin dicha variable ambiental. Además, la experiencia de campo indica que el resto de las agrupaciones no se ajustan a la realidad. En la tabla 2 (forma $\mathrm{R}$ ) se exponen los factores de carga ro- 


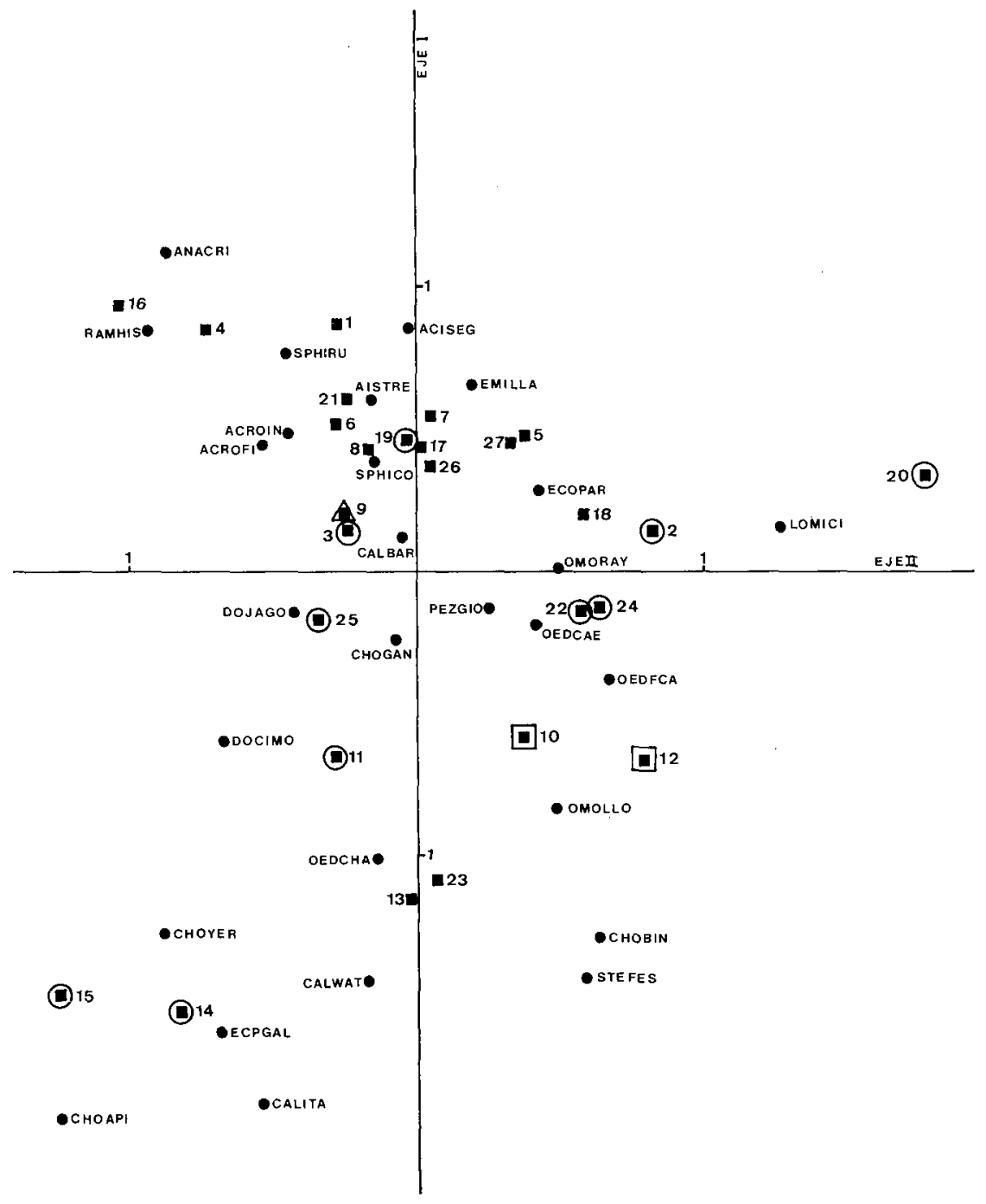

Figura 2. - Representación gráfica de los resultados del análisis de correspondencias de la matriz de 28 especies x 27 muestras, para los ejes I y II

(1) - Pastizales

[- - Matorral almohadillado

- .- Matorrales

A. - Vegetación mixta 
Ejes

Variable

Matorral almohadillado........

Matorral alto claro

Vegetación baja clara

Pastizal no agostante bajo.....

Matorral alto denso

Pastizal no agostante alto......

Pastizal agostante alto..........

\author{
Código
}

MATOAL

MATORO

VEBARA

-

\section{II}

III

PANOBA

0.294

0.728

$-0.337$

MATODE

$-0.492$

0.668

$-0.112$

PANOAL

0.175

$-0.175$

0.815

PATALO

$-0.068$

0.130

0.762

Varianza absorbida

Tabla 1.- Factores de carga rotados por las variables para los tres primeros ejes del análisis binario discriminante en forma «Q» de los 7 tipos de vegetación, de la matriz 51 muestras $\times 30$ especies.

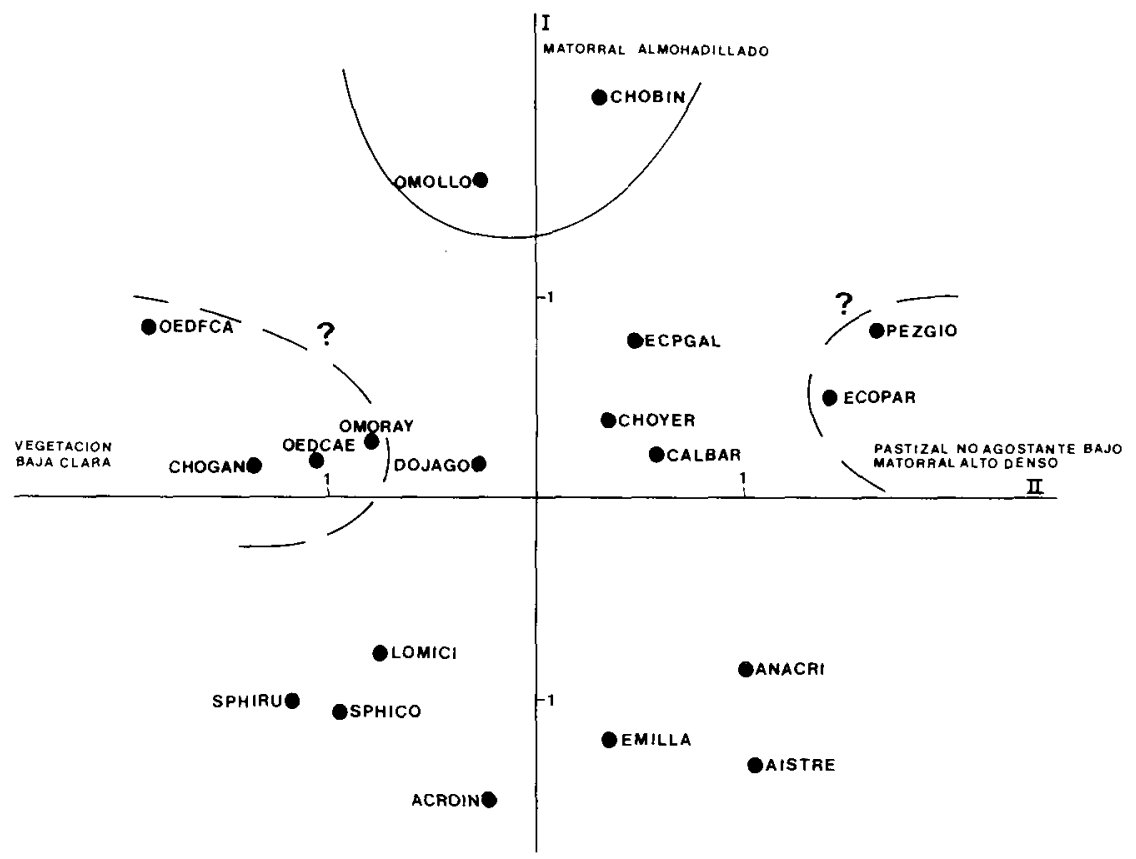

Figura 3.-- Ordenación de las espectes en el plano definido por los dos primeros ejes para el análisis binarie discriminante en forma "Q" de los 7 tipos de vegetación, de la matriz de 51 muestras x 30 especies. 
tados de las 19 especies y los valores de las coordenadas de los tipos de vegetación en los. 5 primeros ejes.

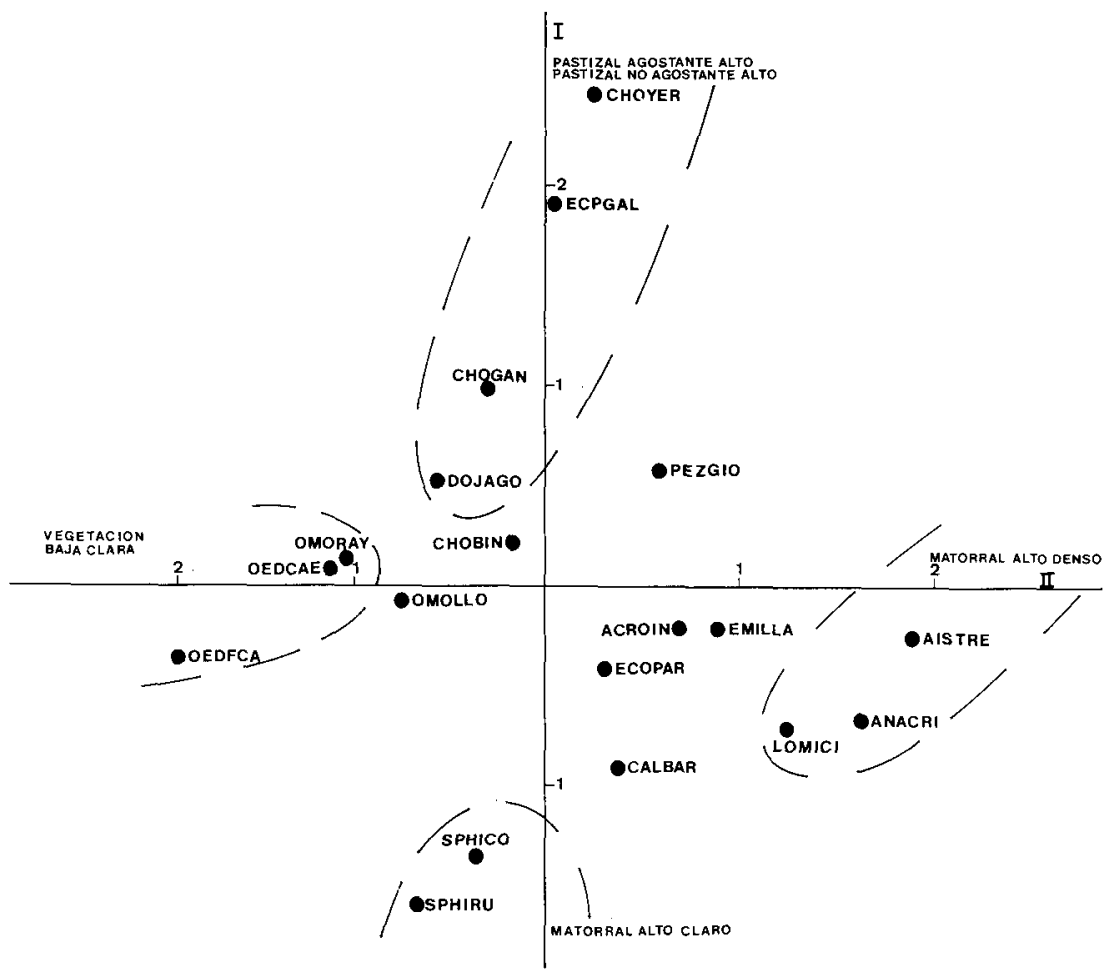

Figura 4.- Ordenación de las especies en el plano definido por los ejes 1 y li para el análisis binario discriminante en forma " $Q$ " de los 6 tipos de vegetación, de la matriz de 46 muestras $\mathrm{x} 30$ especies.

\section{ANALISIS BINARIO DISCRIMINANTE PARA LA VARIABLE TIPOS DE VEGETACION (SOLO SEIS ESTADOS): 30 ESPECIES Y 46 MUESTRAS}

En la tabla 3 se presentan los factores de carga rotados para los tres primeros ejes en forma «Q», de los seis tipos de vegetación. Las figuras 4 y 5 muestran la disposición de las 19 especies que presentaron preferencias marcadas $(p<0,05)$ para un determinado tipo de vegetación en el plano de los ejes I-II y II-III respectivamente. En la tabla 4 se exponen los factores de carga rotados de las especies, varianza absorbida y las coordenadas de las variables ambientales para los cuatro primeros ejes del análisis en forma «R», basado en los tipos de vegetación. 


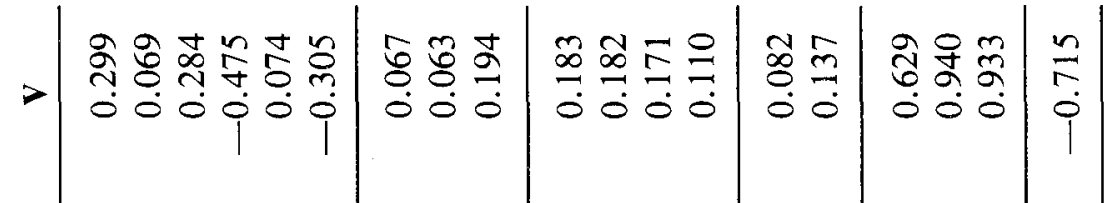

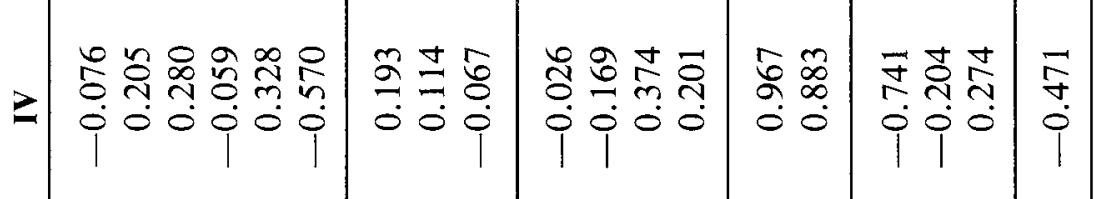

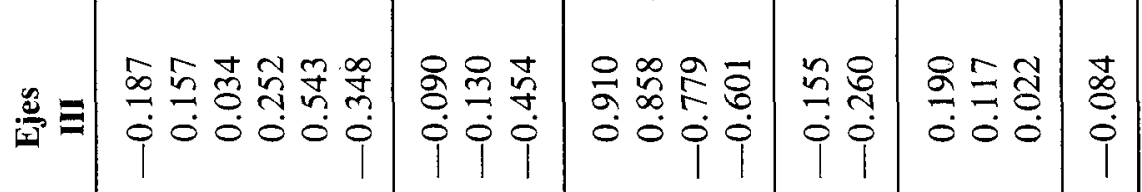

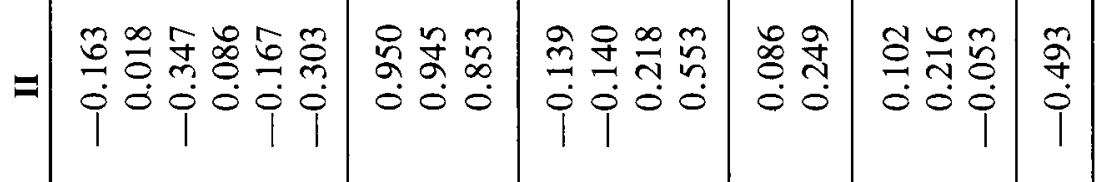

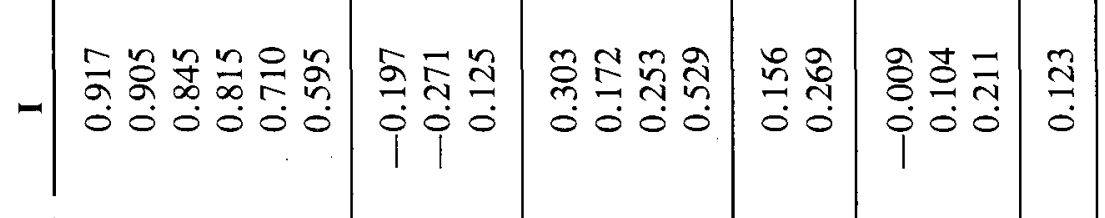

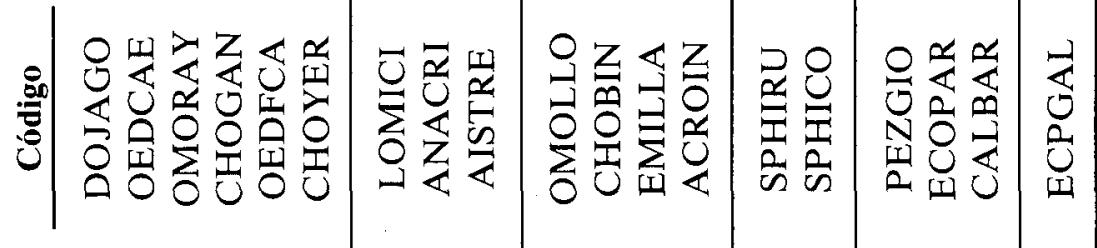

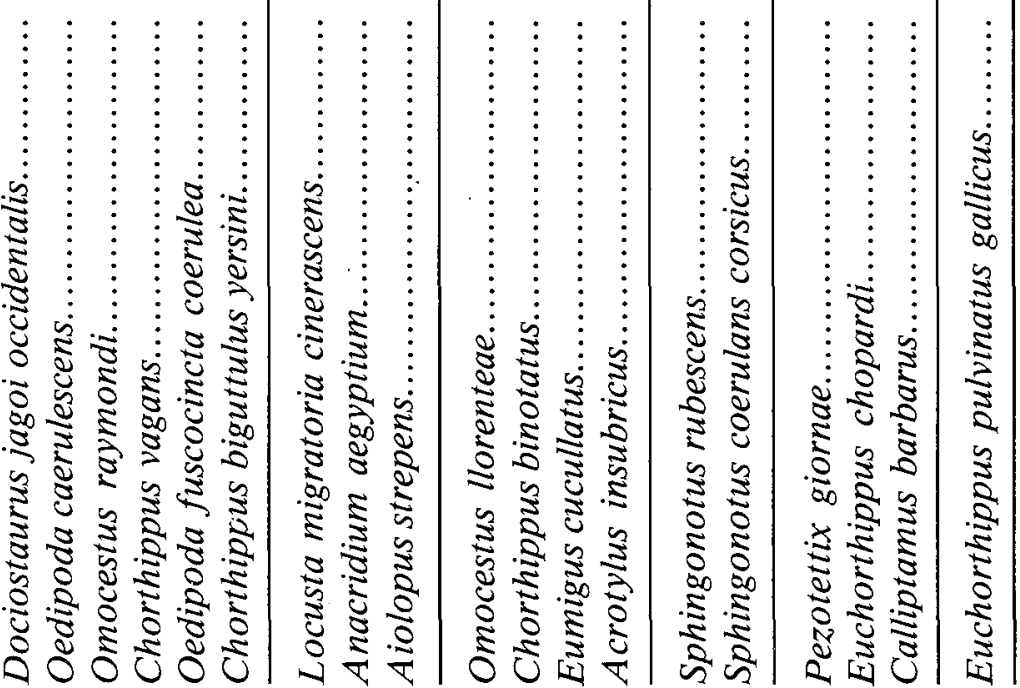



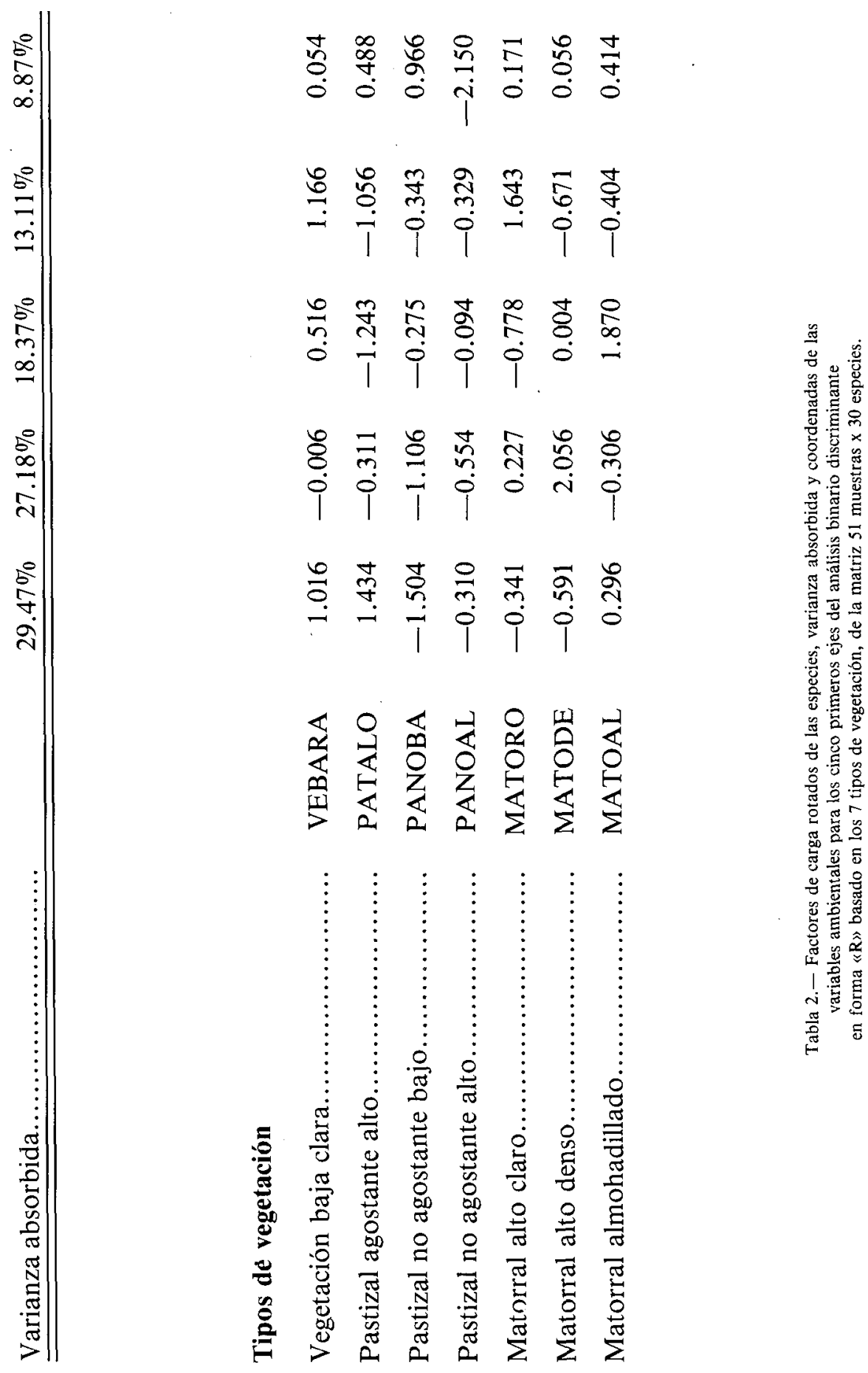
Ejes

Variable

Pastizal no agostante alto.....

Matorral alto claro.

Pastizal agostante alto....

Matorral alto denso

Vegetación baja clara

Pastizal no agostante bajo.....
Código

PANOAL

MATORO

$-0.731$

0.283

$-0.337$

PATALO

0.680

0.103

$-0.033$

MATODE

$-0.248$

0.883

0.031

VEBARA

$-0.430$

$-0.797$

$-0.301$

PANOBA

$-0.044$

0.185

0.946

Varianza absorbida

Tabla 3.- Factores de carga rotados de las variables para los tres primeros ejes del análisis binario discriminante en forma "Q" de los 6 tipos de vegetación, de la matriz 46 muestras $\times 30$ especies.

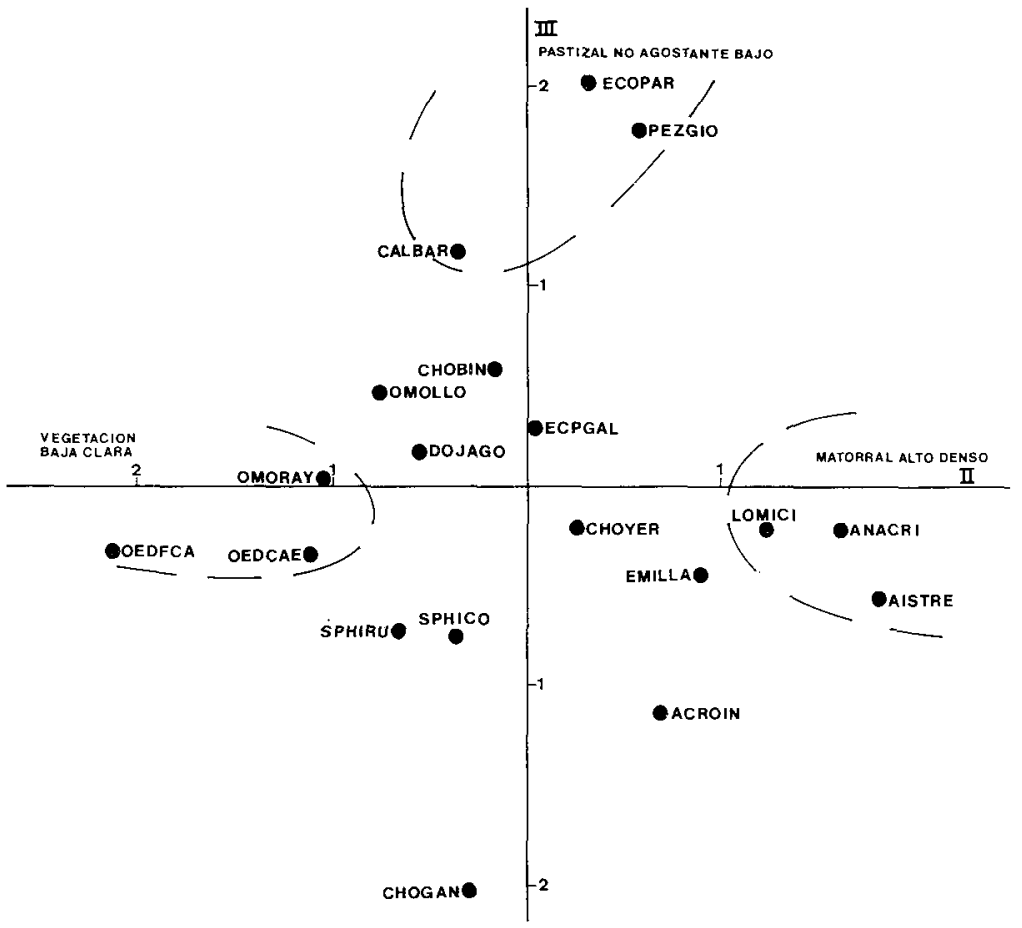

Figura 5.- Ordenación de las especies en el plano definido por los ejes II y III para el análisis binario discriminante en forma "Q⿱ $Q$ » de los 6 tipos de vegetación, de la matriz de 46 muestras x 30 especies. 


\section{DISCUSION Y CONCLUSIONES}

Se han establecido varios grupos de especies con patrones de distribución semejantes en relación con los tipos de vegetación, a partir de los datos proporcionados por los resultados de las formas $\langle Q\rangle y\langle R »$ de los análisis factoriales realizados y de los conocimientos aportados por la experiencia de campo y la bibliografía consultada (grupos cenológicos y ecológicos).

Dentro de cada grupo y, según el grado de caracterización de las distintas poblaciones, se han establecido tres categorías de especies: características (especies que tienden a ser exclusivas de un determinado hábitat); de transición (que no siendo exclusivas tienden a presentarse en más de un tipo de hábitat), e indiferentes (que no presentan preferencias por ningún tipo de hábitat) (PRESA et al., 1983).

Con respecto al estudio de las interaciones especies-tipos de vegetación, pueden establecerse los grupos y relaciones ecológicas que aparecen en la tabla 5.

Hay que destacar el pastizal no agostante bajo, que carece de especies características y de transición. La causa de esta ausencia se debe al escaso número de especies que aparece en este tipo de vegetación dado que este tipo de pastos no puede albergar a los adultos como consecuencia del escaso porte de la vegetación. Esta no resiste el peso de los adultos, pero sí puede acoger a las ninfas sin denotar su presencia. Por tanto, los adultos lo abandonan al no serles útil. De esta manera, este tipo de vegetación resulta poblado, únicamente, por aquellas especies que son constantes en toda la Sierra, resultando, por tanto, indiferentes.

En el caso del pastizal agostante alto tampoco aparece ninguna especie característica.La experiencia de campo indica que Chorthippus biguttulus yersini se muestra en relación con este tipo de vegetación pero que no elude otros, como el pastizal no agostante alto. De esta forma, debe aparecer como especie de transición, no como característica.

El grupo de especies en relación con el matorral alto denso se compone de especies no ampliamente distribuídas, cuya característica común es la de ser extraordinarias voladoras. En el caso de las especies características (Locusta migratoria cinerascens y Anacridium 


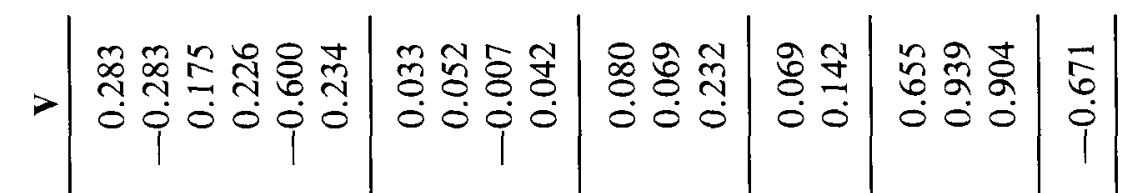

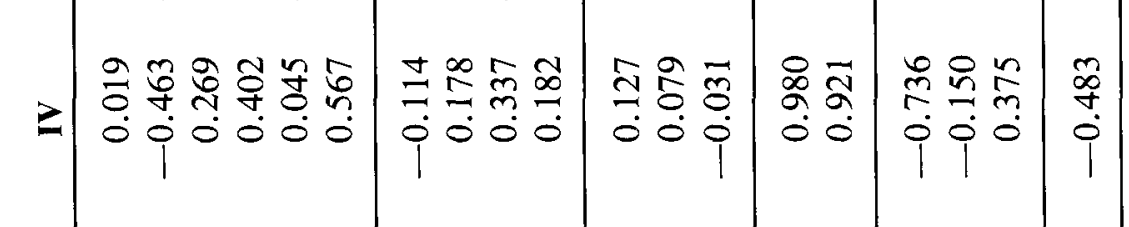

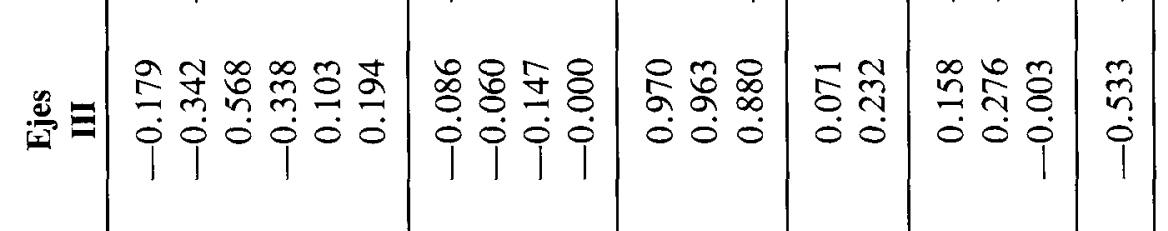

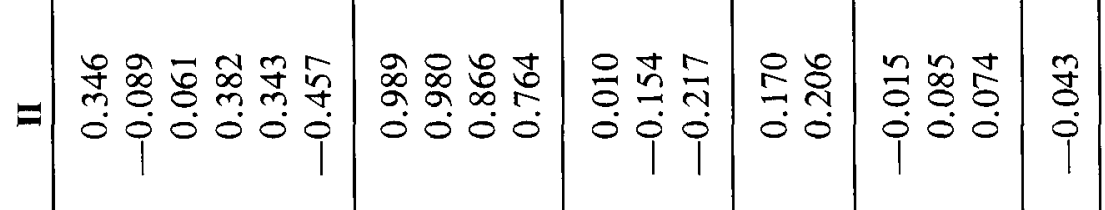

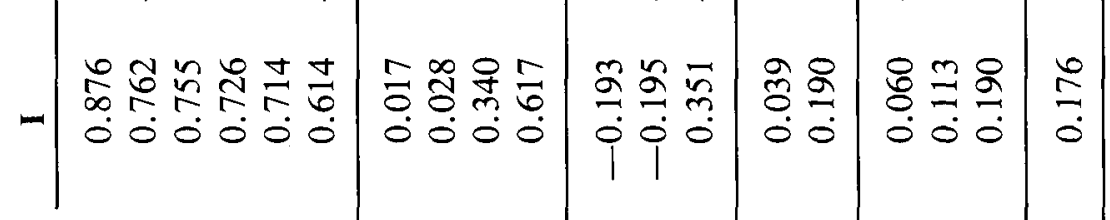

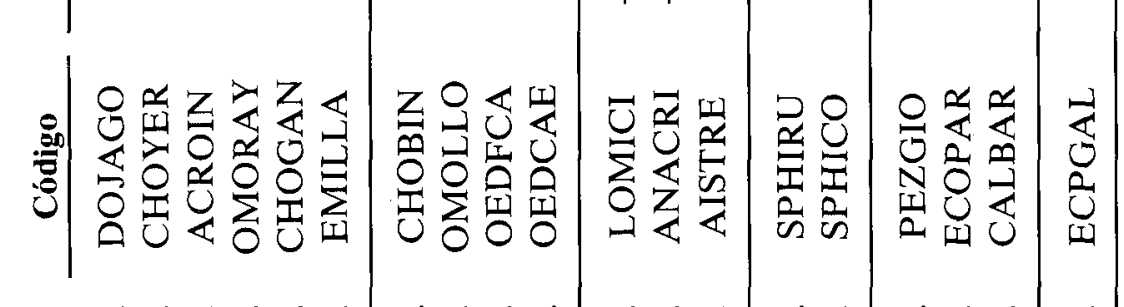

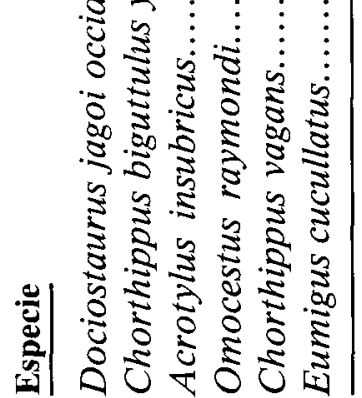

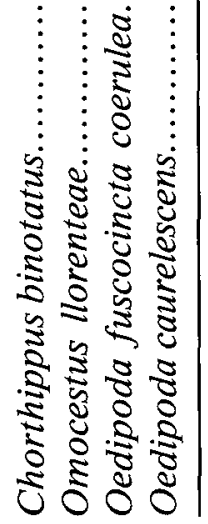

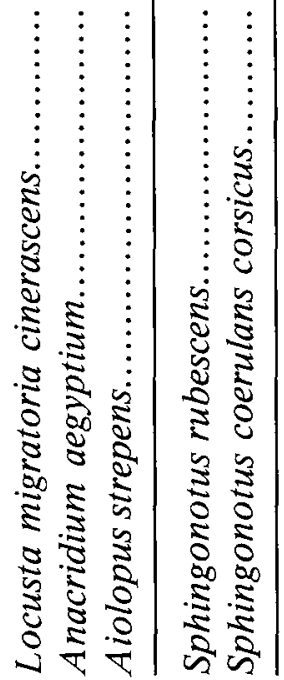

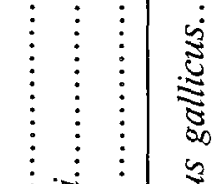



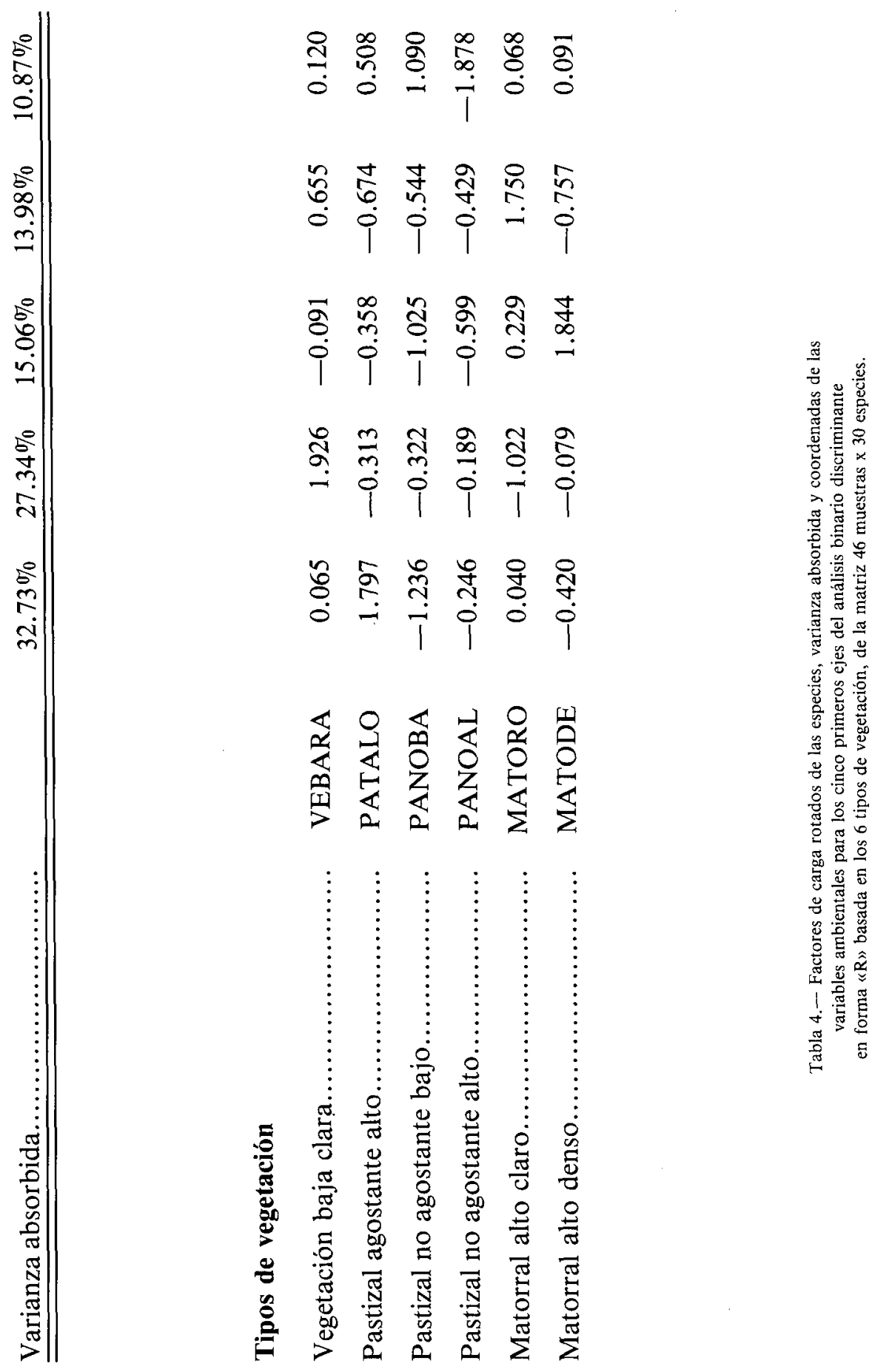


\begin{tabular}{|c|c|c|c|}
\hline & Características & De transición & Indiferentes \\
\hline $\begin{array}{l}\text { Vegetación } \\
\text { baja clara }\end{array}$ & $\begin{array}{l}\text { Oe. fuscocincta } \\
\text { coerulea } \\
\text { Oe. caerulescens }\end{array}$ & $\begin{array}{l}\text { D. jagoi } \\
\text { occidentalis } \\
\text { O. raymondi }\end{array}$ & $\begin{array}{l}\text { Eu. chopardi } \\
\text { C. barbarus } \\
\text { P. giornae } \\
\text { A. insubricus } \\
\text { Eu. cucullatus }\end{array}$ \\
\hline $\begin{array}{l}\text { Pastizal } \\
\text { agostante alto }\end{array}$ & & $\begin{array}{l}\text { A. strepens } \\
\text { Ch. vagans } \\
\text { D. jagoi } \\
\text { occidentalis } \\
\text { Ch. b. yersini } \\
\text { O. raymondi }\end{array}$ & $\begin{array}{l}\text { Eu. chopardi } \\
\text { C. barbarus } \\
\text { P. giornae } \\
\text { A. insubricus } \\
\text { Eu. cucullatus }\end{array}$ \\
\hline $\begin{array}{l}\text { Pastizal no } \\
\text { agostante bajo }\end{array}$ & & & $\begin{array}{l}\text { Eu. chopardi } \\
\text { C. barbarus }\end{array}$ \\
\hline $\begin{array}{l}\text { Pastizal no } \\
\text { agostante alto }\end{array}$ & Eu.p. gallicus & $\begin{array}{l}\text { Ch. vagans } \\
\text { Ch, b. yersini }\end{array}$ & \\
\hline $\begin{array}{l}\text { Matorral } \\
\text { alto claro }\end{array}$ & $\begin{array}{l}\text { Sph. rubescens } \\
\text { Sph. c. corsicus }\end{array}$ & $\begin{array}{l}\text { O. raymondi } \\
\text { Ch. vagans } \\
\text { A. strepens }\end{array}$ & $\begin{array}{l}\text { Eu. chopardi } \\
\text { C. barbarus } \\
\text { A. insubricus } \\
\text { Eu. cucullatus } \\
\text { P. giornae }\end{array}$ \\
\hline $\begin{array}{l}\text { Matorral } \\
\text { alto denso }\end{array}$ & $\begin{array}{l}\text { L. } m \text {. cinerascens } \\
\text { A. aegyptium }\end{array}$ & A. strepens & $\begin{array}{l}\text { Eu. chopardi } \\
\text { Eu. cucullatus } \\
\text { A. insubricus } \\
\text { P. giornae }\end{array}$ \\
\hline $\begin{array}{l}\text { Matorral } \\
\text { almohadillado }\end{array}$ & $\begin{array}{l}\text { Ch. binotatus } \\
\text { O. llorenteae }\end{array}$ & Ch. vagans & $\begin{array}{l}\text { Eu. chopardi } \\
\text { A. insubricus } \\
P . \text { giornae } \\
\text { Eu. cucullatus } \\
\text { C. barbarus }\end{array}$ \\
\hline
\end{tabular}

Tabla 5.- Grupos ecológicos característicos de los tipos de vegetación considerados. 
aegyptium), se trata de especies de gran envergadura que se mimetizan en troncos, buscando siempre vegetación arbustiva, e incluso, arbórea.

El grupo en relación con el matorral almohadillado está formado por dos especies bien distintas: Omocestus llorenteae, braquíptera, que se presenta con gran abundancia en este tipo de vegetación y Chorthippus binonatus, que aparece, con acusada exclusividad, en relación con las matas almohadilladas de Erinacea anthyllis, características de esta vegetación. La relación especie animal-especie vegetal, en este caso, es de tipo trófico, pues es conocida la tendencia a alimentarse de leguminosas de Ch. binotatus.

Abundando en este último tipo de relaciones, merece citarse a la especie $C h$. vagans, especie marcadamente polimórfica que, según las figuras 4 y 5 , se encuentra relacionada con los pastizales. Sin embargo, en el campo se observa que no elude, en absoluto, otros tipos de vegetación y que, en el matorral almohadillado concretamente, mimetiza a Ch. binonatus, adoptando su mismo comportamiento de preferencia exclusiva por Erinacea anthyllis. Así, esta especie aparece como de transición en varios tipos de vegetación.

En cuanto a las especies características de la vegetación baja clara y matorral alto claro, se trata, en ambos casos, de especies marcadamente heliófilas, más si cabe en el segundo caso que en el primero. Son especies que se capturan siempre sobre el suelo, no manifestando en el campo preferencia alguna por ninguna especie vegetal.

En resumen, en este estudio se ha podido establecer este tipo de relaciones especie-medio vegetal de acuerdo con el siguiente esquema.

De esta manera, se confirma que la distribución de las poblaciones de saltamontes depende del tipo fisionómico de la vegetación. Algunas especies, no obstante, se distribuyen preferentemente en relación con su planta nutricia ligándose, de esta manera, a un tipo fisionómico de vegetación. 


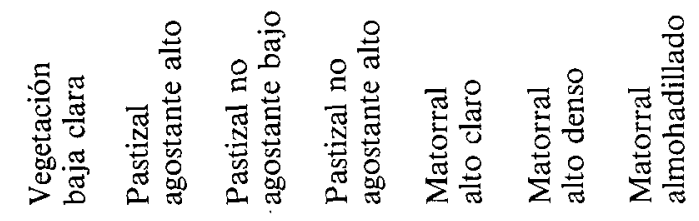

\begin{tabular}{|c|c|c|c|c|c|c|c|}
\hline Oe. fuscocincta coerulea.... & + & & - & - & & - & \\
\hline Oe. caerulescens............. & + & & - & - & & & - \\
\hline Eu. pulvinatus gallicus...... & & & & + & - & - & - \\
\hline Sph. rubescens........ & & - & - & - & + & - & - \\
\hline Sph. coerulans corsicus..... & & - & - & - & + & - & - \\
\hline L. migratoria cinerascens... & & - & - & - & & + & - \\
\hline A. aegyptium... & & - & - & - & & + & - \\
\hline Ch. binotatus.... & - & - & - & - & - & - & + \\
\hline$O . l$ & & & - & - & & & + \\
\hline
\end{tabular}

+ Tiende a estar presente

- Tiende a no estar presente

$\square$ No se elimina la posibilidad de presencia

\section{AGRADECIMIENTOS}

Nuestro más sincero agradecimiento a la Dra. Pilar Martín de Agar por su colaboración en la realización de los programas de ordenador, $y$ al Dr. Francisco Alcaraz por poner a nuestra disposición sus conocimientos botánicos. 


\section{BIBLIOGRAFIA}

BACCETTI, B., (1963). Notulae orthopterologicae XIX. Ricerche sugli Ortotteroidei dell'Appennino ligure orientale per il Centro di Entomologia alpina e Forestale del C.N.R. Redia. 48: 93-163.

CLARIDGE, M.F. \& SINGHRAO, J.S., (1978). Diversity and altitudinal distribution of grasshoppers (Acridoidea) on a Mediterranean mountain. Journ. Biogeugraphy 5: 239-250.

CLARK, E.J., (1947). Studies in the ecology of British grasshoppers. Trans. R. ent. Soc. Lond. 99: $172-222$.

DAHDOUH, B.; DURANTON, J.F. \& LECOQ, M., (1978). Analyse des données sur l'écologie des Acridiens d'Afrique de l'Ouest (Acridiens). Les Cahiers de l'Analyse des Données. 3,4: 459-482.

DIXON, W.J. \& BROWN, M.B., (1979). BMDP-79 Biomedical computer Programs P-series. University of California Press. Berkeley. 880 pp.

DREUX, Ph., (1961). Recherches écologiques et biogéographiques sur les Orthoptères des Alpes françaises. Ann. Sci. Naturelles Zool. 12. ${ }^{2}$ s., 3: 325-760.

DURANTON, J.F. \& LECOQ, M., (1980). Ecology of locusts and grasshoppers (Orthoptera, Acrididae) in Sudanese West Africa. I. Discriminant factors and ecological requirements of acridian species. Acta Oecologica. Oecol. Gener. 1,2: 151-164.

ESTEVE CHUECA, F., (1972). Vegetación y flora de las regiones central y meridional de la provincia de Murcia. I.O.A.T.S. Centro de Edafología y Biología Aplicada del Segura. Murcia. $451 \mathrm{pp}$.

GAJE, S.H. \& MUKERJI, M.K., (1977). A perspective of grasshopper population distribution in Saskatchwan and interrelationship with weather. Environ. Ent., 6(3): 469-479.

GARCIA, M.D., (1983). Estudio faunístico y ecológico de los Acridoidea (Orth., Insecta) de Sierra Espuña (Murcia). Tesis Doctoral. Universidad de Murcia. 284 pp.

LILLO CARPIO, M., (1971). Sierra Espuña. Estudio geográfico. Departamento de Geografía. Universidad de Valencia. $117 \mathrm{ff}$. Tesina de Licenciatura (inédita).

LOPEZ BERMUDEZ, F., (1975). Depósitos de ladera en la Sierra de Espuña (Cordilleras Béticas). Papeles del Departamento de Geografía. 6. Universidad de Murcia: 9-27.

MARTY, R., (1961). Les Orthoptères du Massif du Néouvieille. (Etude systématique et écologique). Bull. Soc. Hist. Nat. Toulouse. 96: 223-240.

MONTES DEL OLMO, C. \& RAMIREZ-DIAZ, L., (1978). Descripción y muestreo de poblaciones y Comunidades vegetales y animales. Publicaciones de la Universidad de Sevilla. Anales de la Universidad Hispalense. Sevilla. $82 \mathrm{pp}$.

NAVARRO ALVARGONZALEZ, A. \& TRIGUEROS MOLINA, E., (1963). Estudio geológico del borde oriental de la Sierra Espuña (Murcia). Not. Com. Inst. Geol. Min. España. 70: 205-210. 
PASCUAL, F., (1978). Estudio preliminar de los Ortópteros de Sierra Nevada. III: Distribución ecológica. Trab. Monogr. Dep. Zool. Univ. Granada (N.S.). 1(2): 65-121.

PEYRE, N. \& PEYRE, Y., (1960). Observaciones geológicas sobre Sierra Espuña (Murcia). Not. Com. Inst. Geol. Min. España. 59: 3-22.

PRESA, J.J.; MONTES, C. \& RAMIREZ-DIAZ, L., (1983). Tipificación de poblaciones de saltamorites (Orth. Acrididae) en relación con la altitud, pisos y tipos de vegetación en la Sierra de Guadarrama (Sistema Central, España). Bol. Asoc. esp. Entom. 6,2: 249-265.

SOUTHWOOD, T.R.E., (1978). Ecological methods with particular reference to the study of insect populations. $2 .^{a}$ ed. Chapman \& Hall.

SOYER, B., (1965). Etude statistique des groupements d'animaux dans les associations végétales des environs de Marseille. IV. Les Orthoptéroïdes (excepté les Embioptères). Bull. Mus. Hist. Nat. Marseille. 25: 57-92.

STRAHLER, A.H., (1978). Binary discriminant analysis: a new method for investigating speciesenvironment relationships. Ecology, 59 (1): 108-116.

VALENZUELA, A., (1963-64). Hidrologia del Norte de Sierra Espuña (Murcia). Anales de la Universidad de Murcia. Filosofía y Letras, 22, 3-4: 139-163.

VOISIN, J.F., (1980). Réflexions à propos d'une méthode simple d'échantillonage des peuplements d'orthoptères en milieu ouvert. Acrida, 9 (4): 159-170. 